\title{
Ivermectin susceptibility and sporontocidal effect in Greater Mekong Subregion Anopheles
}

Kevin C. Kobylinski ${ }^{1} 2^{*}$, Ratawan Ubalee ${ }^{1}$, Alongkot Ponlawat ${ }^{1}$, Chanyapat Nitatsukprasert ${ }^{1}$, Siriporn Phasomkulsolsil ${ }^{1}$, Thanaporn Wattanakul ${ }^{3}$, Joel Tarning ${ }^{3,4}$, Kesara Na-Bangchang ${ }^{5}$, Patrick W. McCardle ${ }^{1,2}$, Silas A. Davidson ${ }^{1,2}$ and Jason H. Richardson ${ }^{1,2,6}$

\begin{abstract}
Background: Novel vector control methods that can directly target outdoor malaria transmission are urgently needed in the Greater Mekong Subregion (GMS) to accelerate malaria elimination and artemisinin resistance containment efforts. Ivermectin mass drug administration (MDA) to humans has been shown to effectively kill wild Anopheles and suppress malaria transmission in West Africa. Preliminary laboratory investigations were performed to determine ivermectin susceptibility and sporontocidal effect in GMS Anopheles malaria vectors coupled with pharmacokinetic models of ivermectin at escalating doses.

Methods: A population-based pharmacokinetic model of ivermectin was developed using pre-existing data from a clinical trial conducted in Thai volunteers at the $200 \mu \mathrm{g} / \mathrm{kg}$ dose. To assess ivermectin susceptibility, various concentrations of ivermectin compound were mixed in human blood meals and blood-fed to Anopheles dirus, Anopheles minimus, Anopheles sawadwongporni, and Anopheles campestris. Mosquito survival was monitored daily for 7 days and a non-linear mixed effects model with probit analyses was used to calculate concentrations of ivermectin that killed $50 \%\left(\mathrm{LC}_{50}\right)$ of mosquitoes for each species. Blood samples were collected from Plasmodium vivax positive patients and offered to mosquitoes with or without ivermectin at the ivermectin $\mathrm{LC}_{25}$ or $\mathrm{LC}_{5}$ for $A$. dirus and An. minimus.

Results: The GMS Anopheles displayed a range of susceptibility to ivermectin with species listed from most to least susceptible being An. minimus $\left(\mathrm{LC}_{50}=16.3 \mathrm{ng} / \mathrm{ml}\right)>$ An. campestris $\left(\mathrm{LC}_{50}=26.4 \mathrm{ng} / \mathrm{ml}\right)=$ An. sawadwongporni $\left(\mathrm{LC}_{50}=26.9 \mathrm{ng} / \mathrm{ml}\right)>$ An. dirus $\left(\mathrm{LC}_{50}=55.6 \mathrm{ng} / \mathrm{ml}\right)$. Mosquito survivorship results, the pharmacokinetic model, and extensive safety data indicated that ivermectin $400 \mu \mathrm{g} / \mathrm{kg}$ is the ideal minimal dose for MDA in the GMS for malaria parasite transmission control. Ivermectin compound was sporontocidal to P. vivax in both An. dirus and An. minimus at the $\mathrm{LC}_{25}$ and $\mathrm{LC}_{5}$ concentrations.

Conclusions: Ivermectin is lethal to dominant GMS Anopheles malaria vectors and inhibits sporogony of P. vivax at safe human relevant concentrations. The data suggest that ivermectin MDA has potential in the GMS as a vector and transmission blocking control tool to aid malaria elimination efforts.
\end{abstract}

Keywords: Ivermectin, Anopheles, Plasmodium, Greater Mekong Sub-region

\footnotetext{
${ }^{*}$ Correspondence: kobylinskikevin@yahoo.com

1 Department of Entomology, Armed Forces Research Institute of Medical

Sciences, 315/6 Rajvithi Road, Bangkok 10400, Thailand

Full list of author information is available at the end of the article
} and indicate if changes were made. The Creative Commons Public Domain Dedication waiver (http://creativecommons.org/ publicdomain/zero/1.0/) applies to the data made available in this article, unless otherwise stated. 


\section{Background}

Artemisinin-resistant Plasmodium falciparum isolates have been identified in the Greater Mekong Subregion (GMS) which threatens to undermine malaria elimination goals [1]. The primary GMS Anopheles vectors of Plasmodium frequently feed outdoors and before people go to sleep $[2,3]$, rendering classical vector control measures such as insecticide-treated bed nets and indoor residual spraying with insecticides less effective. There are currently no vector control tools in the GMS that specifically target outdoor feeding Anopheles which is a major impediment for malaria vector control in the region. Novel vector control tools that target outdoor malaria transmission are urgently needed to support artemisinin resistance containment and malaria elimination efforts in the GMS.

Ivermectin has been shown to be lethal to over a dozen Anopheles species worldwide [4]. Ivermectin mass drug administration (MDA) has been suggested as a possible malaria parasite transmission control tool as it directly targets the vector at the point of human blood feeding. It is one of the few vector control measures that targets outdoor malaria transmission. Laboratory studies $[5,6]$, clinical trials [7-9], and field studies [10,11] demonstrate that ivermectin is lethal to Anopheles gambiae at human relevant concentrations. Ivermectin MDA campaigns in Senegal, Liberia, and Burkina Faso have demonstrated that ivermectin can suppress $P$. falciparum transmission by wild An. gambiae s.l. [11, 12]. In addition to direct mosquito-lethal effects, ivermectin suppresses development of P. falciparum in An. gambiae [13, 14].

Plasmodium transmission in the GMS is complex with numerous Anopheles species serving as primary and secondary vectors throughout the region. Anopheles dirus s.s., found in the GMS east of Myanmar, is a primary malaria vector in forested areas and feeds predominantly outdoors on humans [2, 15]. Anopheles minimus s.s., found throughout the GMS and parts of mainland Asia, is a primary malaria vector outside of forested areas, feeds both indoors and outdoors, and displays a variable feeding preference on humans and cattle across its range $[3,16]$. There are several secondary malaria vectors that may facilitate malaria transmission in the GMS. Anopheles sawadwongporni, a member of the Anopheles maculatus group, has been incriminated as a malaria vector in Thailand [17] and Anopheles campestris, predominantly found in rice paddies throughout the GMS is suspected to be a secondary malaria vector in Thailand [18].

Ivermectin MDA campaigns in West Africa using a 150$200 \mu \mathrm{g} / \mathrm{kg}$ dose were shown to be effective at reducing $P$. falciparum transmission by An. gambiae [11, 12]. However, not all Anopheles may be equally susceptible to ivermectin [4]. This implies that higher doses of ivermectin may be required during MDA to effectively target all Anopheles in a given region. Doses of ivermectin up to $2000 \mu \mathrm{g} / \mathrm{kg}$ were safe and well tolerated in healthy volunteers [19], which is ten times the amount approved for strongyloidiasis treatment in Thailand [20]. The ivermectin dose of $800 \mu \mathrm{g} / \mathrm{kg}$ has been assessed in onchocerciasis-infected patients in an extended trial in Ghana [21, 22] and repeatedly every 3 months in a trial in Cameroon [23]. Adverse events in the ivermectin $800 \mu \mathrm{g} / \mathrm{kg}$ trials may be correlated with the immune response to dead microfilariae and not necessarily linked directly to ivermectin treatment. Trials in healthy and malaria infected patients without concomitant onchocerciasis infection at the ivermectin $800 \mu \mathrm{g} / \mathrm{kg}$ dose are warranted. The lack of adverse events in healthy volunteers at up to ivermectin doses of $2000 \mu \mathrm{g} / \mathrm{kg}$ [19] would support the notion that the adverse events observed in Ghana [21, 22] and Cameroon [23] trials may be linked to the death and clearance of Onchocerca volvulus parasites and not ivermectin toxicity. Several clinical trials have investigated the safety and tolerability of ivermectin at 400 and $800 \mu \mathrm{g} /$ $\mathrm{kg}$, however, to the best of our knowledge, none have assessed the pharmacokinetic properties of ivermectin at these doses, particularly in an Asian population, which is the population of interest in this study.

The mosquito-lethal effects of ivermectin on the GMS malaria vectors $A n$. dirus s.s., An. minimus s.s., An. sawadwongporni, and An. campestris, and the sporontocidal effects of ivermectin on Plasmodium vivax in An. dirus s.s., and An. minimus s.s. were investigated. A population-based pharmacokinetic model of ivermectin and simulated concentration-time profiles after 200, 400 and $800 \mu \mathrm{g} / \mathrm{kg}$ doses was developed, and correlated with mosquito survivorship results to rationally select ivermectin doses for MDA use in the GMS.

\section{Methods}

\section{Pharmacokinetic modelling}

Frequent ivermectin plasma concentrations were collected in 23 healthy Thai volunteers after a standard $200 \mu \mathrm{g} / \mathrm{kg}$ dose [24]. Ivermectin concentrations were transformed into their natural logarithms and characterized using nonlinear mixed-effects modelling in NONMEM version.7.3 (Icon Development Solution, Ellicott City, MD, USA). The first-order conditional estimation method with interaction was used throughout model development. Model diagnostics and automation were performed using Xpose version 4.0 [25], Pirana [26] and Pearl-speaks-NONMEM (PsN; version 3.6.0) [27]. The objective function value (OFV; calculated by NONMEM as proportional to $-2 \times \operatorname{loglikelihood~of~data)~was~used~}$ to discriminate between hierarchical models. A difference between two models $(\Delta \mathrm{OFV})$ of $>3.84$ and $>10.83$ were considered statistically significant at a $\mathrm{P}$ value of 
$<0.05$ and $<0.001$, respectively. Models were assessed using standard goodness-of-fit and simulation-based diagnostics (i.e. numerical and visual predictive checks using 2000 simulations). The robustness of the final model was evaluated by bootstrapping $(n=1000)$.

Different disposition models (one-, two- and three-compartment models) and absorption models (first-order and transit models) were evaluated to describe the pharmacokinetic structural model of ivermectin. Pharmacokinetic parameters were assumed to be log-normally distributed and inter-individual variability was implemented as exponential functions. The effect of body size was evaluated using an allometric function (centred on the medium trial weight of $55 \mathrm{~kg}$ ), scaling clearances with an exponent of $3 / 4$ and volumes with an exponent of 1 . The influence of other individual covariates including gender, age, haemoglobin, haematocrit, total bilirubin, direct bilirubin, alkaline phosphatase, aspartate aminotransferase, and alanine aminotransferase, serum creatinine, blood urea nitrogen, and albumin level were investigated using a stepwise covariate approach ( $P$ value $<0.05$ and $<0.001$ for forward and backward addition and deletion, respectively). The developed final population pharmacokinetic model of ivermectin was used to simulate population mean ivermectin pharmacokinetic concentration-time profiles after a single oral dose of 200, 400 and $800 \mu \mathrm{g} / \mathrm{kg}$ using Berkeley Madonna [28].

\section{Mosquitoes}

All mosquitoes were reared at the Armed Forces Research Institute of Medical Sciences Department of Entomology in Bangkok, Thailand. Anopheles dirus s.s., An. minimus s.s., An. sawadwongporni, and An. campestris were produced as described previously [29]. Adult mosquitoes used for experiments were provided $10 \%$ sucrose solution ad libitum. Mosquitoes were reared at $25 \pm 2{ }^{\circ} \mathrm{C}$ and $80 \pm 10 \%$ relative humidity, and $12 \mathrm{~h}$ light:12 h dark photoperiod. Mosquitoes were between 5 and 8 days post emergence at time of first blood feed, and mosquitoes were sugar starved with access to water from 12 to $18 \mathrm{~h}$ prior to their first blood meal.

\section{Drug}

Powdered formulation of ivermectin compound was obtained from Sigma-Aldrich (St. Louis, USA). Ivermectin was dissolved in dimethylsulfoxide (DMSO) to concentrations of $10 \mathrm{mg} / \mathrm{ml}$ and $20 \mu \mathrm{l}$ aliquots were frozen at $-20{ }^{\circ} \mathrm{C}$. Ivermectin was thawed and serial dilutions were made in phosphate buffered saline (PBS) and $10 \mu \mathrm{l}$ was added to $990 \mu \mathrm{l}$ of blood to reach final concentration desired for mosquito membrane feeding assays. Control blood meals consisted of previously frozen DMSO diluted in PBS to match the ratio of DMSO and PBS fed to mosquitoes in the ivermectin-containing blood meals.

\section{Blood and serum}

Pooled whole blood collected in 350-ml citrate phosphate dextrose adenine (CPDA-1) anticoagulant bags (National Blood Centre, Thai Red Cross Society, Bangkok, Thailand) was used to maintain colonies and perform ivermectin lethal concentration experiments. Blood was never more than 2 weeks post collection at the time of mosquito blood feeds. Plasmodium vivax-infected blood was drawn from individual volunteers into 10 -ml sodium heparin tubes (NH) (158 USP units, BD Vacutainer, Franklin Lakes, NJ, USA) for the ivermectin sporogony experiments. Serum (type $\mathrm{AB}+$ ) from malaria-naïve persons (Interstate Blood Bank Inc, Memphis, TN, USA) was processed and stored frozen at $-20^{\circ} \mathrm{C}$ until ready for use in ivermectin sporogony experiments.

\section{Ivermectin lethal concentration calculations}

Anopheles dirus, An. minimus, An. sawadwongporni, and An. campestris were blood fed multiple concentrations of ivermectin to determine the lethal concentration that killed 50\% $\left(\mathrm{LC}_{50}\right), 25 \%\left(\mathrm{LC}_{25}\right)$ and $5 \%\left(\mathrm{LC}_{5}\right)$ of the mosquitoes at day 7 post blood meal, following previous methods $[5,13]$. Control blood meals consisted of DMSO diluted in PBS to match the concentration found in the highest ivermectin treatment group in each replicate. After blood feeding via a membrane feeder, unfed mosquitoes were removed and discarded. Blood-fed mosquitoes were held in 3.5-1 plastic containers with access to $10 \%$ sucrose and kept in an incubator at $25 \pm 1{ }^{\circ} \mathrm{C}$ and $70 \pm 10 \%$ relative humidity, on a $12 \mathrm{~h}$ light: $12 \mathrm{~h}$ dark photoperiod. Mosquito survivorship was monitored for 7 days, every $24 \mathrm{~h}$ dead mosquitoes were removed and recorded, and on day 7 all remaining mosquitoes were frozen and counted as alive.

The time above the $\mathrm{LC}_{50}$-values for different species of mosquitoes was determined from the simulated pharmacokinetic concentration-time profiles after a single oral dose of 200, 400 and $800 \mu \mathrm{g} / \mathrm{kg}$, as described above.

\section{Effect of ivermectin on Plasmodium vivax sporogony}

Previously it was demonstrated that the ivermectin $\mathrm{LC}_{25}$ inhibited the development of P. falciparum in An. gambiae [13], and a similar experimental design was repeated here. Mosquitoes reared in Bangkok were transported to a field laboratory at the Mae Sot Malaria Medical Clinic in Mae Sot, Tak Province. Plasmodium vivaxinfected blood was collected from malaria-infected patients reporting to malaria clinics operated by the Thailand Ministry of Public Health in Tak Province (protocol WRAIR\#1949A). Patient plasma was removed and replaced by centrifuging blood samples at $3000 \mathrm{rpm}$ for 5 min, plasma removed and blood washed with RPMI twice, and malaria-naïve $\mathrm{AB}+$ serum was added at a 
50:50 ratio [30]. One millilitre of blood was fed to each group of 100 mosquitoes in 0.5-1 cardboard containers and unfed mosquitoes were removed. Mosquitoes were maintained on $10 \%$ sucrose ad libitum at the Mae Sot field insectary $\left(25 \pm 1{ }^{\circ} \mathrm{C}\right.$ and $70 \pm 10 \%$ relative humidity, light $12 \mathrm{~h}: 12 \mathrm{~h}$ dark photoperiod). Once infected with $P$. vivax, blood-fed mosquitoes were securely transported back to Bangkok and maintained in a separate insectary $\left(25 \pm 1{ }^{\circ} \mathrm{C}\right.$ and $70 \pm 10 \%$ relative humidity, light $12 \mathrm{~h}: 12 \mathrm{~h}$ dark photoperiod).

For An. dirus, four experimental feeding schedules performed where ivermectin $\mathrm{LC}_{25}$ and $\mathrm{LC}_{5}$ was blood fed concomitantly with $P$. vivax at day post infection zero (DPI 0), ivermectin $\mathrm{LC}_{5}$ three days before (DPI -3$) P$. vivax, and ivermectin $\mathrm{LC}_{25}$ six (DPI 6) or nine (DPI 9) after $P$. vivax (Fig. 1). For An. minimus only one experimental feeding schedule was performed when ivermectin $\mathrm{LC}_{25}$ and $\mathrm{LC}_{5}$ was blood fed concomitantly with $P$. vivax (DPI 0). For each mosquito species and feeding schedule there was an ivermectin $\left(\mathrm{LC}_{25}\right.$ or $\left.\mathrm{LC}_{5}\right)$ and control group, which had matching concentrations of DMSO and PBS. When mosquitoes were fed ivermectin or control meals at DPI $-3,6$ or 9, uninfected donor blood at a $50 \%$ haematocrit was used for the blood feed. Unfed mosquitoes were removed from the feeding containers after each blood meal. Mosquitoes from the DPI 0 and DPI -3 feeding schedules were dissected 7 days post parasite ingestion to enumerate oocysts. Midguts were dissected with minuten pins into saline on a microscope slide and stained with $0.4 \%$ mercurochrome and viewed at $40 \times$ magnification with a compound microscope to determine oocyst prevalence and intensity. Approximately 25 mosquitoes were dissected from each control and treatment group at each dissection time point. Dissectors were blinded to treatment groups. Due to lack of initial mosquito blood feeding and mosquitoes lost to

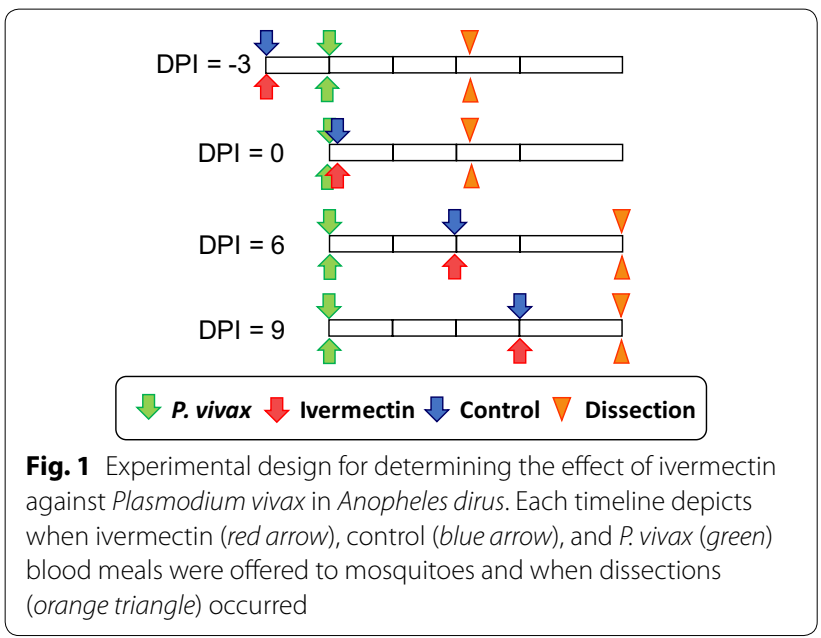

experimental mortality and oocyst dissections at day 7 post parasite feeding, not enough mosquitoes remained at day 14 post parasite feeding to determine sporozoite prevalence for the DPI -3 and 0 experiments. Mosquitoes from the DPI 6 and 9 experiments were dissected 14 days post parasite ingestion to determine sporozoite prevalence. Salivary glands were dissected with minuten pins into saline on a microscope slide and viewed at $40 \times$ magnification to determine sporozoite prevalence.

\section{Effect of Plasmodium vivax infection on Anopheles dirus survivorship response to ivermectin}

Previously it was shown that An. gambiae infected with $P$. falciparum were slightly but significantly more susceptible to an ivermectin blood meal at day 14 post parasite ingestion [13]. Anopheles dirus were fed a P. vivax blood meal or a non-infectious blood meal prepared from uninfected donor blood and serum was replaced as described above. Both blood meals were spiked with ivermectin at the $\mathrm{LC}_{25}$. After blood feeding, 50 blood fed females from each treatment group were transferred to a new 0.5-1 cardboard carton. Mosquito survivorship was monitored daily and dead mosquitoes were removed from the containers until day 14 post blood meal. Any live mosquitoes at day 14 were frozen and counted as alive. A separate group of mosquitoes were fed a $P$. vivax control blood meal to ensure that the $P$. vivax sample was infectious to mosquitoes and only isolates that successfully infected at least $80 \%$ of $A n$. dirus were included in the results.

\section{Statistical analysis}

A non-linear mixed effects model with probit analysis was used to calculate $\mathrm{LC}_{50}, \mathrm{LC}_{25}$ and $\mathrm{LC}_{5}$ values with Statistical Analysis Software (SAS Institute Inc, Cary, NC, USA) as described previously [5]. Hazard ratios for mosquito mortality at day 7 post blood meal were calculated using Poisson regression analysis with STATA version 12.1 (StataCorp LLC, College Station, TX, USA). Oocyst and sporozoite prevalence (i.e. proportion of infected mosquitoes) was compared by Fishers Exact test. Oocyst intensity (i.e. number of oocysts per infected mosquito) was compared by the Mann-Whitney U test. To determine if $P$. vivax infection altered mosquito survivorship post-ivermectin ingestion survival data were analysed by the Mantel-Cox method. The Fishers Exact, MannWhitney U, and survival analysis data were analysed with Prism 7 (GraphPad Software Inc, San Diego, CA, USA).

\section{Results}

Pharmacokinetic modelling

A total of 534 venous blood samples were collected from 23 healthy volunteers (12 males and 11 females) for up to 7 days after a single oral dose of $200 \mu \mathrm{g} / \mathrm{kg}$ of ivermectin. 
There were no observations reported to be below the lower limit of quantification. Observed ivermectin concentrations were best described by a two-compartment disposition model, with no further improvement of an additional disposition compartment $(\triangle \mathrm{OFV}=0)$. A flexible transit absorption model $(\mathrm{n}=2)$ improved the model fit substantially compared to the first-order absorption $(\Delta \mathrm{OFV}=-378)$. The implementation of body weight as an allometric function had a negligible impact on model fit $(\triangle \mathrm{OFV}=0.516)$, but it was retained in the final model based on the strong biological prior. Implementing body weight as a covariate in the model also allowed for translation and interpretation to other study populations. No additional covariates had a significant impact on the model fit. The final model showed satisfactory goodness of fits (Additional file 1: Figure S1) and simulation-based predictive performance (Fig. 2). A numerical predictive check resulted in $2.62 \%$ (95\% CI $0.37-6.18 \%$ ) and $0.56 \%$ (95\% CI $0.56-5.43 \%)$ of ivermectin observations below and above the simulated $95 \%$ prediction interval, respectively. Ivermectin population pharmacokinetic parameter estimates and secondary parameters from final model are summarized in Table 1. The simulated concentration-time profiles after a single oral dose of 200, 400 and $800 \mu \mathrm{g} / \mathrm{kg}$ of ivermectin are illustrated in Fig. 3.

\section{Ivermectin lethal concentration calculations}

A total of 11,622 mosquitoes were used to calculate the concentration of ivermectin that killed: An. dirus (reps $=6, \mathrm{n}=5029$ ), An. minimus (reps $=6, \mathrm{n}=2376$ ),

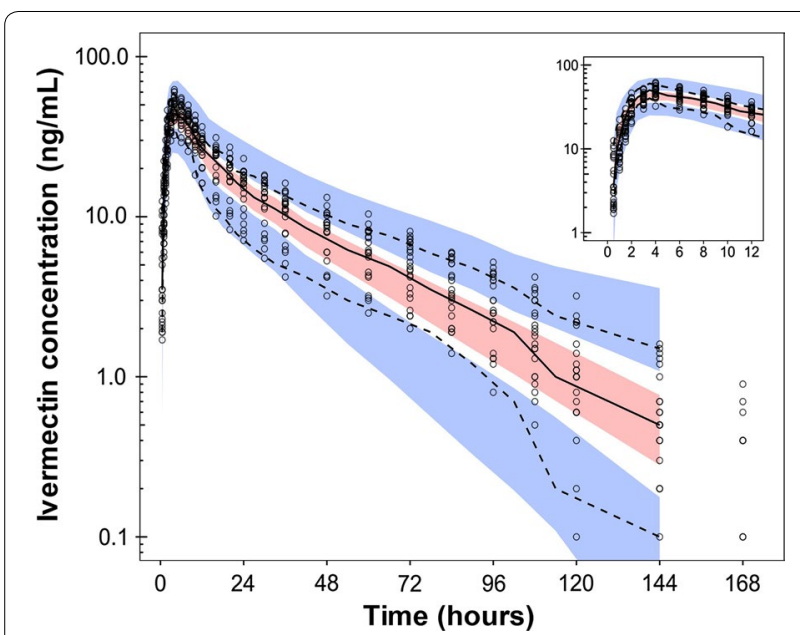

Fig. 2 Visual predictive check of final population pharmacokinetic model of ivermectin in healthy volunteers. Open circles represent observed concentrations; solid and dashed lines represent the 5th, 50th, and 95th percentiles of the observed data; shaded areas represent the $95 \%$ confidence intervals of the simulated 5th, 50th and 95th percentiles $(n=2000)$
An. sawadwongporni (reps $=4, \mathrm{n}=1446$ ), and An. campestris (reps $=4, \mathrm{n}=2786$ ). Ivermectin compound at human-relevant concentrations reduced the survivorship of all GMS Anopheles species investigated (Fig. 4). The GMS Anopheles displayed a range of susceptibility to ivermectin with species listed from most to least susceptible being An. minimus $>$ An. campestris $=$ An. sawadwongporni $>A n$. dirus (Table 2). The amount of time that ivermectin compound was predicted to be above each Anopheles species 7-day- $\mathrm{LC}_{50}$ concentration in human plasma for each 200, 400 and $800 \mu \mathrm{g} / \mathrm{kg}$ dose is reported in Table 3. For An. dirus all ivermectin concentrations had a significantly increased hazard of mortality compared to the control group; for An. minimus all ivermectin concentrations had significantly increased hazard of mortality compared to the control group except for 6,3 and $2 \mathrm{ng} / \mathrm{ml}$; for An. sawadwongporni all ivermectin concentrations had significantly increased hazard of mortality compared to the control group except for $12,10,8,6$, 5 and $4 \mathrm{ng} / \mathrm{ml}$; and for An. campestris all ivermectin concentrations had significantly increased hazard of mortality compared to the control group except for 10, 6, 5 and $3 \mathrm{ng} / \mathrm{ml}$ (Additional file 1: Table S1).

\section{Effect of ivermectin on Plasmodium vivax sporogony}

When co-ingested at DPI 0 , ivermectin reduced the development of $P$. vivax in both $A n$. dirus and $A n$. minimus. In $A n$. dirus the $P$. vivax oocyst prevalence was reduced at DPI 0 at the ivermectin $\mathrm{LC}_{25}$ by 44.7\% $\left(\chi^{2}=29.52, P<0.0001\right.$, reps $\left.=6, \mathrm{n}=285\right)$ and $\mathrm{LC}_{5}$ reduced by $33.6 \%\left(\mathrm{x}^{2}=17.9, P<0.0001\right.$, reps $=6$, $\mathrm{n}=300$ ) (Fig. 5a) and mean oocyst intensity was reduced at the $\mathrm{LC}_{25}$ by $51.2 \%(P=0.0022, \mathrm{n}=156)$ and $\mathrm{LC}_{5}$ by 24.7\% ( $P=0.0389, \mathrm{n}=178)$ (Fig. 5b). In An. minimus the $P$. vivax oocyst prevalence was reduced at DPI 0 at the ivermectin $\mathrm{LC}_{25}$ by $58.8 \%\left(\chi^{2}=21.72, P<0.0001\right.$, reps $=5, \mathrm{n}=172)$ and $\mathrm{LC}_{5}$ by $31.3 \%\left(\mathrm{X}^{2}=12.93\right.$, $P=0.0004$, reps $=5, \mathrm{n}=235$ ) (Fig. 6a) and mean oocyst intensity was reduced at the $\mathrm{LC}_{25}$ by $28.5 \%(P=0.1018$, $\mathrm{n}=60)$ and $\mathrm{LC}_{5}$ by $35.3 \%(P<0.0001, \mathrm{n}=139)$ (Fig. $\left.6 \mathrm{~b}\right)$. Note, the lack of significance for oocyst intensity for $A n$. minimus at the ivermectin $\mathrm{LC}_{25}$ is due to mosquito mortality and sporontocidal effect reducing oocyst prevalence contributing to too few mosquitoes to dissect in the treatment group to determine significance.

However, ivermectin did not significantly alter $P$. vivax infection in $A n$. dirus when given at DPI $-3,6$ or 9 . Plasmodium vivax oocyst prevalence decreased slightly in $A n$. dirus at DPI -3 at the $\mathrm{LC}_{5}$ by $3.9 \%\left(\mathrm{x}^{2}=0.12\right.$, $P=0.4313$, reps $=3, \mathrm{n}=150$ ) (Fig. 7) and oocyst intensity increased by $24.2 \%(P=0.4405, \mathrm{n}=100)$ (Additional file 1: Figure S2). Plasmodium vivax sporozoite prevalence in An. dirus decreased slightly at DPI 6 at the $\mathrm{LC}_{25}$ 
Table 1 Parameter estimates from final population pharmacokinetic model of ivermectin in healthy volunteers

\begin{tabular}{|c|c|c|c|c|}
\hline Parameter & Population estimates (\%RSE) & $95 \% \mathrm{Cl}^{\mathrm{b}}$ & IIV [\%CV] (\%RSE) & $95 \% \mathrm{Cl}^{\mathrm{b}}$ \\
\hline $\mathrm{F}$ & 1 (fixed) & - & $10.7(42.9)$ & $4.82-14.1$ \\
\hline$k_{a}\left(h^{-1}\right)$ & $0.317(3.97)$ & $0.296-0.343$ & - & - \\
\hline MTT (h) & $0.496(17.1)$ & $0.350-0.679$ & $56.1(36.2)$ & $31.9-77.4$ \\
\hline$C L / F(I / h)$ & $9.02(5.49)$ & $8.08-10.1$ & $23.1(25.2)$ & $15.3-27.5$ \\
\hline$V_{C} / F(I)$ & $115(4.38)$ & $106-125$ & - & - \\
\hline $\mathrm{Q} / \mathrm{F}(\mathrm{l} / \mathrm{h})$ & $16.2(5.34)$ & $14.7-18.1$ & - & - \\
\hline$V_{p} / F(I)$ & $157(6.59)$ & $139-178$ & $22.8(36.0)$ & $13.5-29.9$ \\
\hline$\sigma$ & 0.0361 & $0.0263-0.0463$ & - & - \\
\hline \multicolumn{5}{|l|}{ Secondary parameters ${ }^{c}$} \\
\hline Terminal half-life (h) & $25.0(23.7-29.5)$ & & & \\
\hline $\mathrm{AUC}_{0-168}(\mathrm{ng} \times \mathrm{h} / \mathrm{ml})$ & 1331 (919-1406) & & & \\
\hline$C_{\max }(\mathrm{ng} / \mathrm{ml})$ & $45.7(40.3-46.9)$ & & & \\
\hline $\mathrm{T}_{\max }(\mathrm{h})$ & $4.76(4.67-5.04)$ & & & \\
\hline
\end{tabular}

$F$ relative bioavailability, $k_{a}$ absorption rate constant, $M T T$ mean transit absorption time, $C L / F$ apparent oral elimination clearance, $V_{C} / F$ apparent volume of distribution of central compartment, $Q / F$ apparent inter-compartmental clearance, $V_{p} / F$ apparent volume of distribution of peripheral compartment, $\sigma$ variance of the residual variability

a Population mean values and inter-individual variability (IIV) were estimated by NONMEM. The coefficient of variation (\%CV) for IIV was calculated as $100 \times \sqrt{e^{\text {estimate }}-1}$

b The relative standard error (\%RSE) was calculated as $100 \times\left(\frac{\mathrm{SD}}{\text { Mean value }}\right)$ from the non-parametric bootstrap results $(\mathrm{n}=1000)$. The $95 \%$ confidence interval $(95 \%$ Cl) is presented as the $2.5-97.5$ percentiles of the bootstrap estimates

c Post hoc parameter estimates from final pharmacokinetic model of ivermectin presented as median (interquartile range)

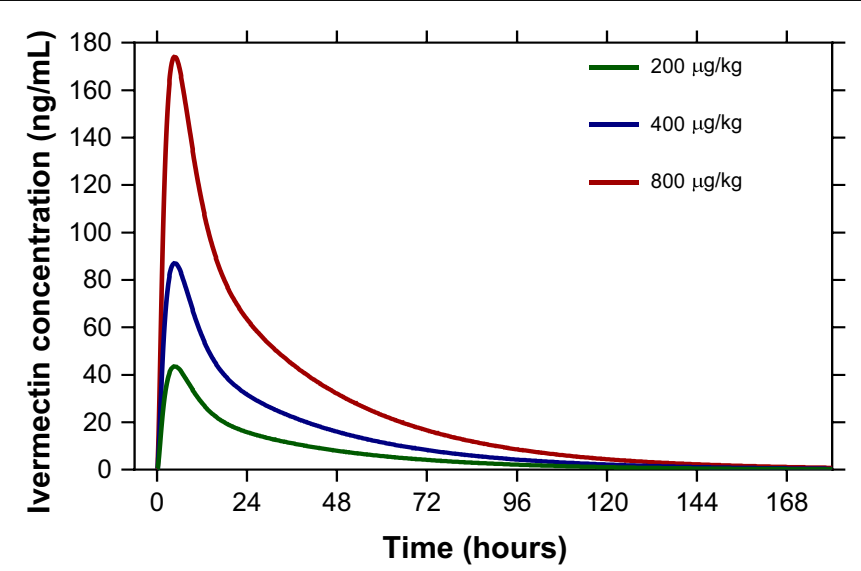

Fig. 3 Simulation population mean pharmacokinetic profiles of ivermectin at single oral doses of 200, 400 and $800 \mu \mathrm{g} / \mathrm{kg}$, based on final population pharmacokinetic model

by $4.5 \%\left(\chi^{2}=0.11, P=0.7751\right.$, reps $\left.=4, \mathrm{n}=195\right)$ and increased slightly at DPI 9 at the $\mathrm{LC}_{25}$ by $4.4 \%\left(\chi^{2}=0.08\right.$, $P=0.8872$, reps $=4, \mathrm{n}=200$ ) (Fig. 7).

\section{Effect of Plasmodium vivax infection on Anopheles dirus survivorship response to ivermectin}

Plasmodium vivax infection did not alter survivorship of $A n$. dirus that co-ingested parasites and ivermectin $\mathrm{LC}_{25}$ on DPI $0\left(x^{2}=2.83, P=0.0925\right.$, reps $\left.=4, \mathrm{n}=400\right)$ compared to control mosquitoes that only ingested ivermectin $\mathrm{LC}_{25}$ (Fig. 8).

\section{Discussion}

All four Anopheles species investigated were susceptible to ivermectin at concentrations predicted to be present in humans following oral administration. This suggests that ivermectin MDA has a potential role in malaria elimination in the GMS as it is a novel vector control tool that could directly combat outdoor malaria transmission. Anopheles dirus and An. minimus are the two most important primary vectors of malaria in the GMS while An. campestris and An. sawadwongporni are potential secondary vectors [31]. Since An. dirus is arguably the most 

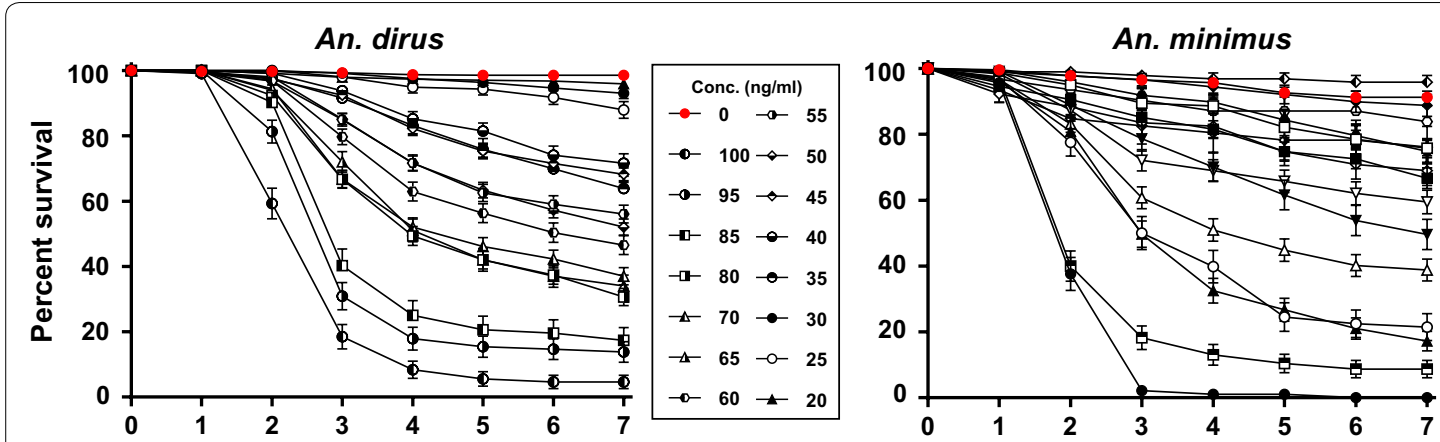

Conc. (ng/ml)

$0 \rightarrow-10$

$\rightarrow 30 \rightarrow-8$

$\rightarrow 28 \rightarrow 6$

$\multimap 25 \multimap 5$

$\leftarrow 20 \rightarrow 4$

$\triangle 18-3$

$+15 \rightarrow 2$

$\rightarrow 12 \leadsto 1$

An. sawadwongporni

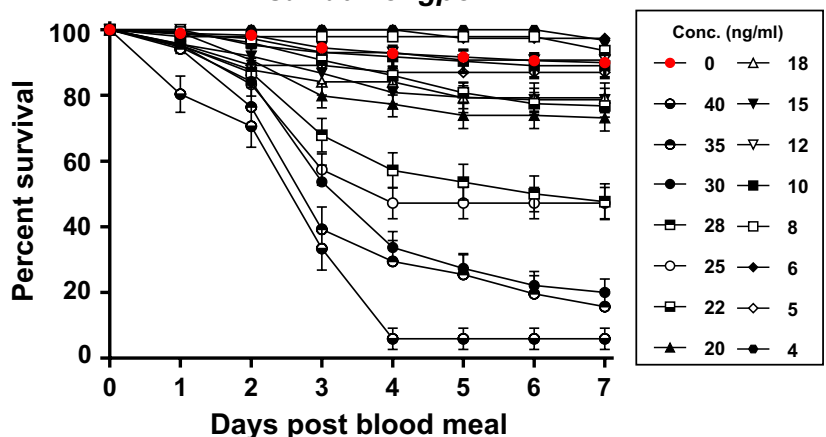

An. campestris

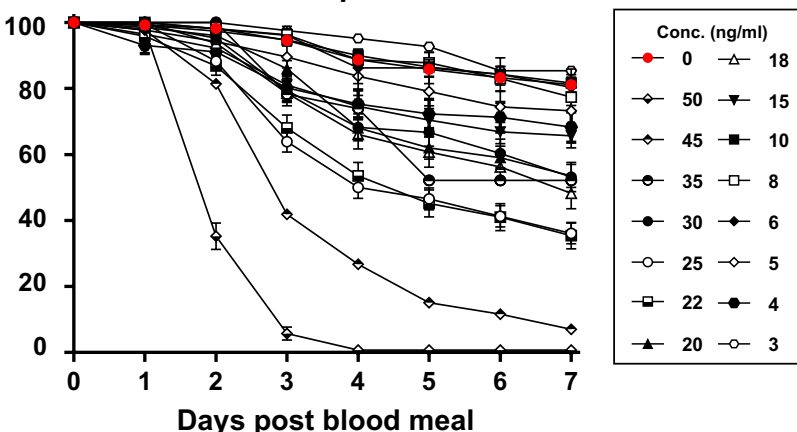

Fig. 4 Anopheles survival post ingestion of ivermectin compound by day. Boxed legends represent the concentrations of ivermectin imbibed by each species. Not all concentrations included in the lethal concentration analyses are displayed here. Each line represents 1-6 replicates with standard error

Table 2 Lethal concentration values by Anopheles species at 7 days post blood meal

\begin{tabular}{|c|c|c|c|c|c|c|}
\hline & $\mathrm{LC}_{50}$ & $95 \% \mathrm{FL}^{*}$ & $\mathrm{LC}_{25}$ & $95 \% \mathrm{FL}$ & $\mathrm{LC}_{5}$ & $95 \% \mathrm{FL}$ \\
\hline An. dirus & 55.6 & {$[52.3-59.1]$} & 38.1 & {$[34.4-41.3]$} & 22.1 & [18.1-25.6] \\
\hline An. minimus & 16.3 & [11.6-19.4] & 11.3 & {$[5.7-14.5]$} & 6.7 & [1.9-10.1] \\
\hline An. sawadwongporni & 26.9 & {$[24.8-28.8]$} & 21.8 & [18.6-23.8] & 16.1 & [11.9-18.8] \\
\hline An. campestris & 26.4 & {$[21.9-30.5]$} & 18.9 & [13.9-22.6] & 11.7 & {$[6.9-15.5]$} \\
\hline
\end{tabular}

${ }^{*} F L$ 95\% fiducial limits

Table 3 Time (in days) above each Anopheles species 7-day- $\mathrm{LC}_{50}$ by dose as predicted by the pharmacokinetic model

\begin{tabular}{llll}
\hline Dose & $\mathbf{2 0 0} \mathbf{~} \mathbf{g} / \mathbf{k g}$ & $\mathbf{4 0 0} \mathbf{\mu g} / \mathbf{k g}$ & $\mathbf{8 0 0} \mathbf{~} \mathbf{g} \mathbf{k g}$ \\
\hline An. dirus & $\mathrm{n} / \mathrm{a}$ & 0.4 & 1.1 \\
An. minimus & 0.9 & 1.9 & 3 \\
An. sawadwongporni & 0.4 & 1.2 & 2.2 \\
An. campestris & 0.5 & 1.2 & 2.3 \\
\hline
\end{tabular}

important GMS malaria vector it is critical that ivermectin MDA deliver a dose of ivermectin high enough to control this vector. Population peak ivermectin concentrations after a single oral dose of $200 \mu \mathrm{g} / \mathrm{kg}$ did not reach the 7 -day- $\mathrm{LC}_{50}$ of $A n$. dirus. However, pharmacokinetic modelling and simulation presented here suggest that a single oral ivermectin dose of 400 or $800 \mu \mathrm{g} / \mathrm{kg}$ results in concentrations that surpasses the ivermectin 7-day- $\mathrm{LC}_{50}$ for $A n$. dirus (Table 3). Based on the An. dirus in vitro survivorship results, pharmacokinetic model output and extensive safety data discussed below, the ivermectin $400 \mu \mathrm{g} / \mathrm{kg}$ dose appears to be the ideal minimal dose to administer during MDA for malaria control in the GMS.

The simulated time above the $A n$. dirus ivermectin 7 -day- $\mathrm{LC}_{50}$ at ivermectin doses of $400 \mu \mathrm{g} / \mathrm{kg}(0.4$ days) or $800 \mu \mathrm{g} / \mathrm{kg}$ (1.1 days) would not seem to deliver a substantial mosquito-killing window following ivermectin 

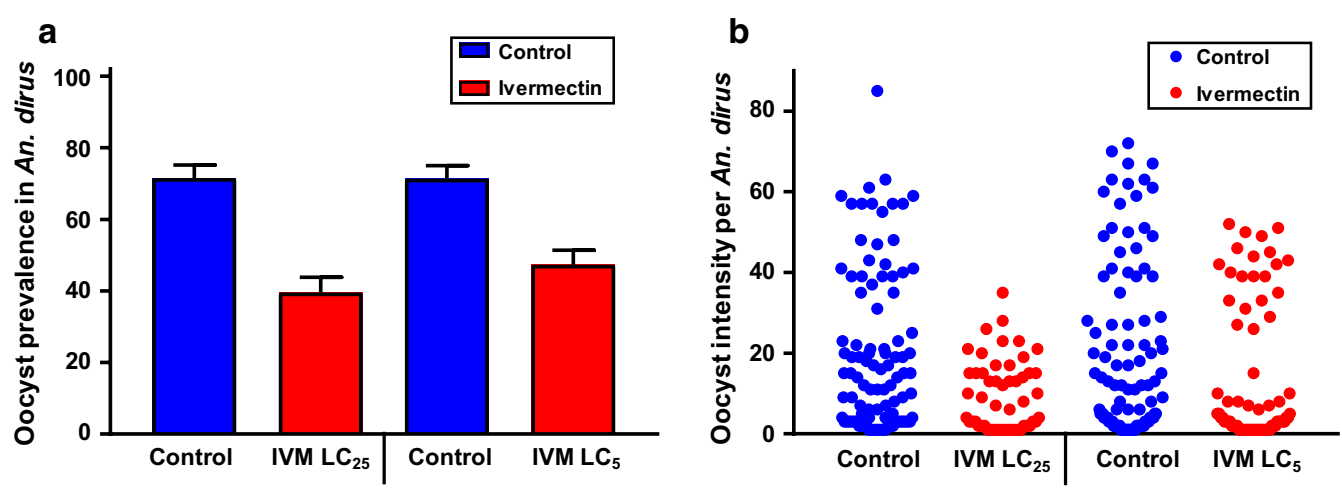

Fig. 5 Plasmodium vivax oocyst prevalence (a) and intensity (b) in Anopheles dirus when ivermectin co-ingested with parasites. Plasmodium vivax oocyst prevalence (a) and intensity $(\mathbf{b})$ in An. dirus when ivermectin $\mathrm{LC}_{25}(38.1 \mathrm{ng} / \mathrm{ml})$ and $\mathrm{LC}_{5}(22.1 \mathrm{ng} / \mathrm{ml})$ co-ingested with parasites at DPI 0 . Oocyst prevalence was significantly reduced at the $\mathrm{LC}_{25}$ and $\mathrm{LC}_{5}$ concentrations as determined by the Fishers Exact test. Oocyst intensity was significantly reduced at the $\mathrm{LC}_{25}$ and $\mathrm{LC}_{5}$ concentrations as determined by the by the Mann-Whitney $\mathrm{U}$ test. Prevalence error bars represent standard error
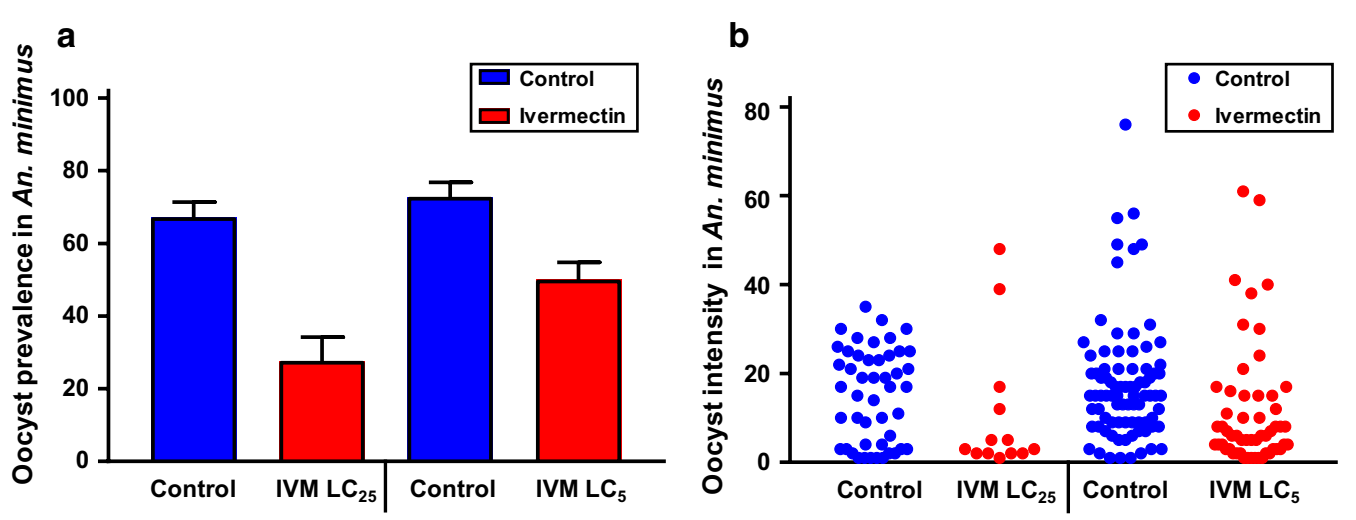

Fig. 6 Plasmodium vivax oocyst prevalence (a) and intensity (b) in Anopheles minimus when ivermectin co-ingested with parasites. Plasmodium vivax oocyst prevalence (a) and intensity $(\mathbf{b})$ in $A n$. minimus when ivermectin $\mathrm{LC}_{25}(11.3 \mathrm{ng} / \mathrm{ml})$ and $\mathrm{LC}_{5}(6.7 \mathrm{ng} / \mathrm{ml})$ co-ingested with parasites at DPI 0. Oocyst prevalence was significantly reduced at the $L C_{25}$ and $L C_{5}$ concentrations as determined by the Fishers Exact test. Oocyst intensity was significantly reduced at the $\mathrm{LC}_{5}$ but not the $\mathrm{LC}_{25}$ concentration as determined by the by the Mann-Whitney $\mathrm{U}$ test. Prevalence error bars represent standard error

MDA. However, it should be noted that the $200 \mu \mathrm{g} /$ $\mathrm{kg}$ dose would only provide a simulated 0.6 days above the An. gambiae 5-day- $\mathrm{LC}_{50}$ value of $22.4 \mathrm{ng} / \mathrm{ml}$ [5], and yet ivermectin MDAs in Senegal at doses of $150 \mu \mathrm{g} /$ $\mathrm{kg}$ substantially reduced An. gambiae 5-day survivorship by $43.6 \%$ for up to 6 days post MDA [10]. Furthermore, the pharmacokinetic model predicts that only $0.432 \mathrm{ng} / \mathrm{ml}$ of ivermectin parent compound would be present in a typical patient of $55 \mathrm{~kg}$ body weight 6 days post MDA at the $150 \mu \mathrm{g} / \mathrm{kg}$ dose, which is well below the concentration capable of killing An. gambiae. This clearly illustrates that in vitro mosquito membrane feeds and pharmacokinetic predictions of parent compound likely underestimate the full mosquito-lethal potential of ivermectin-treated humans. One possible explanation may be that ivermectin produces active mosquito-lethal in vivo metabolites with different pharmacokinetic properties that extend the duration of mosquito-lethal effect beyond that of the parent compound. Human liver microsomes have been used to characterize some of the ivermectin metabolites [32]. A small $(\mathrm{n}=4)$ mass balance study in humans determined that mean peak plasma concentration of metabolites was 2.5 -fold greater than that of the parent compound and the effective half-lives of the metabolites was approximately 2.9 days while the parent compound half-life was $11.8 \mathrm{~h}$ [33]. Further attention to the characterization of ivermectin mosquitolethal metabolites is warranted, especially in light of the novel long-lasting ivermectin formulations in development for human use [34-36]. 


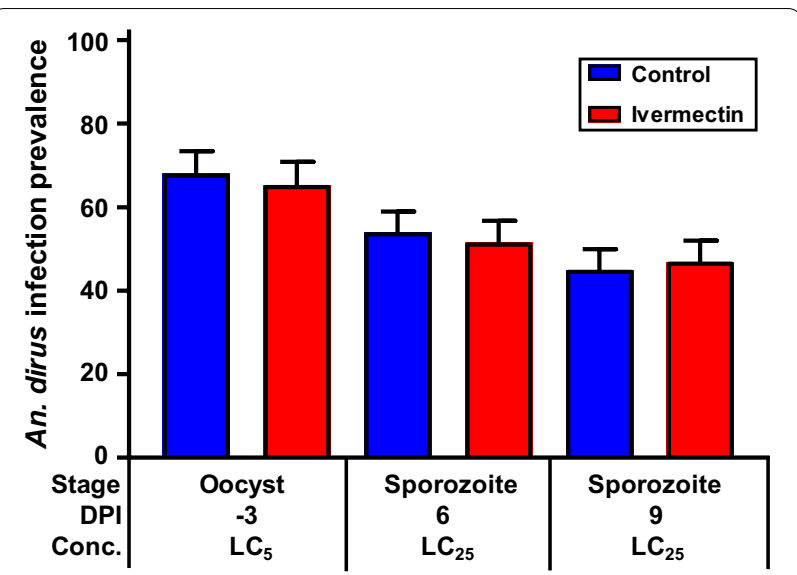

Fig. 7 Plasmodium vivax infection prevalence in Anopheles dirus when ivermectin ingested at DPI $-3,6$, and 9. Plasmodium vivax infection prevalence in An. dirus when ivermectin $L_{5}(22.1 \mathrm{ng} / \mathrm{ml})$ ingested at DPI -3 and $\mathrm{LC}_{25}(38.1 \mathrm{ng} / \mathrm{ml})$ ingested at DPI 6 and 9 . Oocyst prevalence was not significantly reduced at the DPI $-3,6$, or 9 time points as determined by the Fishers Exact test. Prevalence error bars represent standard error

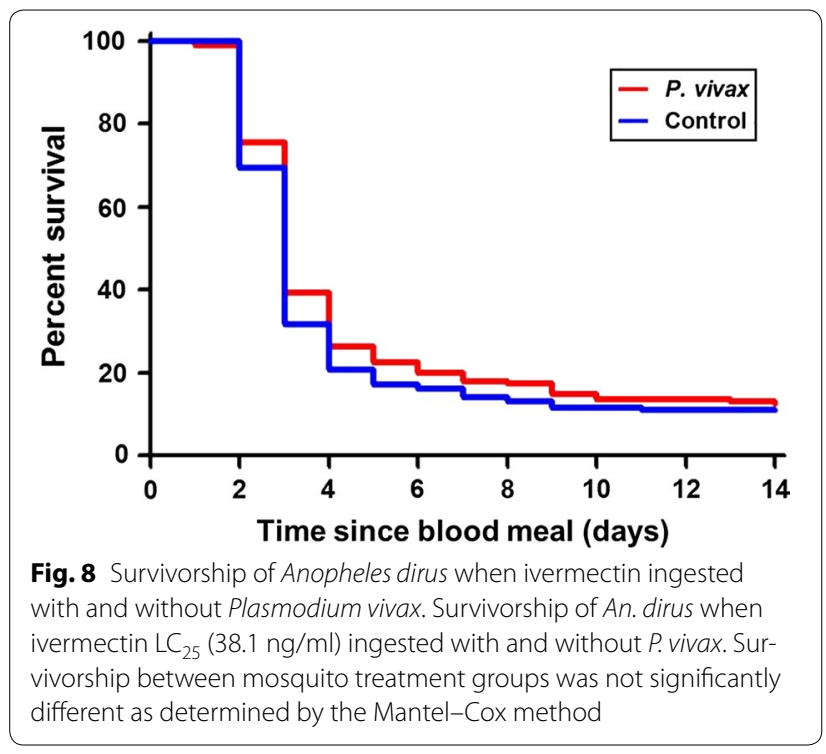

The final population pharmacokinetic model developed in this study described ivermectin concentrations in healthy volunteers satisfactorily. Overall parameter estimates were similar to that reported previously in healthy volunteers and patients with onchocerciasis [37, 38]. Simulation-based diagnostics demonstrated a good predictive performance, which suggests that the developed model is suitable to use for simulations. However, the model was developed on data from a single oral dose of $200 \mu \mathrm{g} / \mathrm{kg}$ and extrapolations beyond that (i.e. simulations of 400 and $800 \mu \mathrm{g} / \mathrm{kg}$ doses) assumes that ivermectin shows dose linearity at this dosing range [19]. Body weight, implemented as allometric function, produces a biologically plausible covariate relationship [39, 40] between ivermectin exposure and body weight so that the developed pharmacokinetic model can be used to simulate other populations at risk, such as children. However, the pharmacokinetic properties of a drug can be very different in children and adults due to the rapid change in body size, organ function, body composition, and enzyme maturation, which occur during the early years of life. Prospective clinical trials are urgently needed in children since there are currently no pharmacokinetic assessments of ivermectin in children and adolescents below 17 years of age. A previous study [8] reported that female participants and participants with higher body mass index (BMI) had higher day-7 ivermectin concentrations. However, BMI was not found to be a significant covariate on any pharmacokinetic parameters in the current analysis, perhaps due to the relatively narrow range of BMI $\left(17.8-22.8 \mathrm{~kg} / \mathrm{m}^{2}\right)$ studied here. Simulation of ivermectin concentration-time profiles at different dosing regimens were also performed in a previous study [41]. The authors obtained pharmacokinetic parameters from the literature, based on American healthy volunteers and assuming a 30\% inter-individual variability in each parameter. Their simulated concentration-time profile after a single dose of $800 \mu \mathrm{g} / \mathrm{kg}$ of ivermectin resulted in an average peak concentration of $108.1 \mathrm{ng} / \mathrm{ml}$ [41], which is somewhat lower than the value simulated using the model developed here $(174 \mathrm{ng} / \mathrm{ml})$.

The ivermectin dose of $400 \mu \mathrm{g} / \mathrm{kg}$ has been investigated in thousands of adults and children, in healthy and infected persons (e.g. lymphatic filariasis, onchocerciasis, loaisis, ascariasis, trichuriasis, hookworm and lice), in more than 20 clinical trials in ten countries, including India [42], Cameroon [23], Ghana [43], Gabon [44], Sri Lanka [45-47], Mali [48], Papua New Guinea [49, 50], French Polynesia [51-58], Brazil [59-62], Haiti [63, 64], and France [65]. Repeated ivermectin administration at doses of $400 \mu \mathrm{g} / \mathrm{kg}$ was safe in two trials in adults who were treated every two weeks for 12 weeks in Sri Lanka [45] and Brazil [61]. The ivermectin dose of $400 \mu \mathrm{g} / \mathrm{kg}$ was deemed safe enough to perform several rounds of MDA to thousands of people in India [42], Cameroon [23], Papua New Guinea [49], and French Polynesia [56, 57] with minimal adverse events reported. Ramaiah and colleagues led the largest MDA trial of ivermectin $400 \mu \mathrm{g} / \mathrm{kg}$ study to date, wherein five entire villages, roughly 10,000 people, including children and adults of both gender, were treated by MDA nine times over an 11-year period. The $400 \mu \mathrm{g} / \mathrm{kg}$ ivermectin dose is now recommended for lymphatic filariasis when twice yearly ivermectin $200 \mu \mathrm{g} / \mathrm{kg}$ MDA cannot logistically be 
performed [66]. This extensive safety data should justify the use of ivermectin $400 \mu \mathrm{g} / \mathrm{kg}$ for MDA in the GMS.

The sporogony experiments conducted here clearly indicate a sporontocidal effect of ivermectin against P. vivax in both An. dirus and An. minimus (Figs. 5, 6). Both $P$. vivax oocyst prevalence and intensity were significantly reduced when ivermectin compound at the $\mathrm{LC}_{25}$ and $\mathrm{LC}_{5}$ when ingested concomitantly (DPI 0) with parasites in An. dirus and An. minimus. A previous study found that ivermectin compound significantly reduced $P$. falciparum prevalence in An. gambiae when ingested concomitantly (DPI 0 ) at the ivermectin $\mathrm{LC}_{25}$ but not the $\mathrm{LC}_{5}$, and had no effect on oocyst intensity at either concentration [13]. A sporontocidal effect would reduce onward transmission from infected persons that received ivermectin MDA while gametocytaemic. This may be more relevant for transmission suppression for $P$. vivax than $P$. falciparum as $P$. vivax gametocytes mature much more rapidly and are therefore present before people become ill enough to seek treatment [67]. These studies only assess the sporontocidal effect of ivermectin in the mosquito, future studies should investigate the potential gametocytocidal action of ivermectin.

There was no effect of ivermectin $\mathrm{LC}_{5}$ compound on P. vivax oocyst prevalence (Fig. 7) or intensity (Additional file 1: Figure S2) when ingested by An. dirus at DPI -3 . This is in direct contrast to previous findings which showed ivermectin compound $\mathrm{LC}_{5}$ at DPI -3 reduced oocyst prevalence of $P$. falciparum in An. gambiae [13]. Ingestion of blood from ivermectin-treated cattle 4 days before ingestion of field isolates of $P$. falciparum from infected patients did not have a sporontocidal effect [68]. Ivermectin $\mathrm{LC}_{25}$ compound failed to reduce $P$. vivax sporozoite prevalence in $A n$. dirus when ingested at DPI 6 or 9 (Fig. 7), while there was a significant sporontocidal effect at the ivermectin $\mathrm{LC}_{25}$ at DPI 6 and 9 for P. falciparum in An. gambiae [13]. This indicates that ivermectin can have differential sporontocidal impact with different Anopheles and Plasmodium species combinations.

Previously it was suggested that ivermectin sporontocidal effect may be due to direct effects on the mosquito, specifically ivermectin delaying or altering formation of the peritrophic matrix [13]. Indeed, ivermectin has been shown to delay and alter peritrophic matrix formation in Aedes aegypti [69], delay blood meal digestion in An. gambiae [5], and upregulates peritrophic matrix gene expression following ivermectin ingestion in An. gambiae [70]. However, limited investigations by two other laboratories were unable to identify a sporontocidal effect of ivermectin against P. falciparum NF54 strain in An. gambiae or Anopheles stephensi [8] or with field isolates of P. falciparum in An. gambiae [71]. This suggests more complex interaction of ivermectin on mosquito and parasite interaction, possibly by influencing mosquito midgut microbiota. The mosquito midgut microbiota composition has recently been shown to dramatically alter Anopheles immune response and thus Plasmodium infection [72]. Original investigations of avermectin, the biological precursor of ivermectin, suggested no direct effect on a range of bacteria species [73]. However, more recent evidence indicates that avermectin can inhibit growth of Staphylococcus aureus [74], and ivermectin can inhibit growth of Chlamydia trachomatis [75] and Mycobacterium tuberculosis [76]. This recent antibacterial evidence suggests that ivermectin may influence mosquito midgut microbiota composition, which could in turn alter Plasmodium infection. Recently, the midgut microbiota composition was shown to significantly alter formation of the peritrophic matrix [77], thus possible alteration of the midgut microbiota by ivermectin may influence formation of the peritrophic matrix and in turn alter Plasmodium infection. Since different insectaries around the world likely have different midgut bacteria microbiota compositions in colonized mosquitoes, this may explain the differences observed by different laboratories when investigating ivermectin sporontocidal effects. Much remains to be explored to determine the sporontocidal mode of action of ivermectin.

Concomitant ingestion of $P$. vivax and ivermectin $\mathrm{LC}_{25}$ failed to alter $A n$. dirus survivorship compared to ivermectin $\mathrm{LC}_{25}$ alone (Fig. 8). This in contrast to the finding that $P$. falciparum-infectious $A n$. gambiae that ingested ivermectin $\mathrm{LC}_{25}$ at DPI 14 were significantly, albeit modestly, more susceptible to ivermectin compared to uninfected An. gambiae [13]. There were some caveats to the $P$. vivax and An. dirus survival study in that mosquitoes had to be transported between and housed in two different insectaries and P. vivax blood was collected freshly from a single donor in sodium heparin tubes and uninfected blood from multiple donors was stored briefly in CPDA-1 bags. There was no significant difference in $A n$. dirus survivorship when fed a control or ivermectin $\mathrm{LC}_{25}$ meal mixed with blood collected in CPDA-1 or sodium heparin tubes (Additional file 1: Figure S3, additional information text). Future investigations are warranted to determine whether Plasmodium infection in Anopheles alters susceptibility to ivermectin.

During ivermectin MDAs for malaria, numerous neglected tropical diseases (NTDs) in the GMS would be affected, including lymphatic filariasis, scabies, lice, gnathostomiasis, and soil-transmitted helminths (STHs) such as strongyloidiasis, ascariasis and trichuriasis. It has been estimated that $50 \%$ of persons in resource-poor communities in the GMS have one or more STH [78], and strongyloidiasis afflicts between 40 to $60 \%$ of persons in rural Cambodia [79] and Laos [80]. Ivermectin was found to be very effective at treating strongyloidiasis 
and repeated MDAs would further benefit afflicted communities as re-infection rates in Cambodia can be quite high [81]. Years of experience with ivermectin MDA for onchocerciasis in Africa have demonstrated that treated persons clearly recognize and appreciate the secondary benefits that ivermectin treatment has on NTDs [82].

MDA with artemisinin combination therapy (ACT) is being piloted in the GMS [1] and Africa [83]. One problem facing ACT MDA is that asymptomatic persons may not perceive a direct personal benefit of clearing malaria parasites. However, if ivermectin were incorporated into ACT MDA then this may improve compliance as persons could observe a direct personal benefit to MDA participation by reducing NTD burdens. Ivermectin and ACT MDA for malaria control, when rationally deployed in targeted hotspot areas of active Plasmodium transmission, would act in concert by clearing infected persons of their malaria parasites while reducing the transmission potential of the extant mosquito population. This could essentially reset malaria transmission when the next wave of naïve Anopheles emerge from the larval habitat and feed on a population cleared of their malaria parasites. Modelling suggests that if ivermectin is added to anti-malarial drug MDA this will reduce the number of MDAs and time required to achieve elimination [84]. The combination of ivermectin and artemether-lumefantrine was shown to be very safe [8]. Two clinical trials are currently being conducted to assess the safety, tolerability, pharmacokinetics and mosquito-lethal efficacy of ivermectin and dihydroartemisinin-piperaquine in Thailand (NCT02568098) and Kenya (NCT02511353). If ivermectin can integrate with ACT MDA, then this could become a powerful new tool for malaria elimination.

\section{Conclusions}

Key malaria vectors in the GMS are susceptible to ivermectin at human relevant concentrations. Ivermectin is sporontocidal to $P$. vivax in An. dirus and An. minimus. A population pharmacokinetic model was developed for ivermectin after a single oral dose administration of $200 \mu \mathrm{g} /$ $\mathrm{kg}$ in healthy volunteers. The developed model described observed data adequately and was used to simulate population mean concentration-time profiles after 200, 400 and $800 \mu \mathrm{g} / \mathrm{kg}$ doses of ivermectin. Mosquito in vitro survivorship results and pharmacokinetic model results indicate that the ivermectin dose of $400 \mu \mathrm{g} / \mathrm{kg}$ is the ideal minimal dose to administer during MDA in the GMS. There may be active ivermectin metabolites that extend the duration of mosquito-lethal effect beyond what is predicted by parent compound concentrations. Ivermectin MDA could be a powerful new tool to combat outdoor malaria transmission and assist malaria elimination efforts in the GMS.

\section{Additional file}

Additional file 1. Supplemental information for pharmacokinetic model goodness of fit, mosquito infection and mosquito survival output.

\section{Abbreviations}

ACT: artemisinin combination therapy; BMl: body mass index; CPDA: citrate phosphate dextrose adenine; DMSO: dimethylsulfoxide; DPI: day post infection; GMS: Greater Mekong Sub-region; $\mathrm{LC}_{50}$ : lethal concentration that kills 50\% of mosquitoes; MDA: mass drug administration; NTDs: neglected tropical diseases; OFV: objective function value; PBS: phosphate buffered saline; STHs: soil-transmitted helminths.

\section{Authors' contributions}

KCK designed the experiments, KCK and CN conducted the experiments, KCK and PWM analyzed the data, TW and JT performed the pharmacokinetic modelling, RU and AP provided the reagents, KCK, RU, AP, CN, SP, TW, JT, KN, PWM, SAD, and JHR wrote the manuscript. All authors read and approved the final manuscript.

\section{Author details}

${ }^{1}$ Department of Entomology, Armed Forces Research Institute of Medical Sciences, 315/6 Rajvithi Road, Bangkok 10400, Thailand. ${ }^{2}$ Entomology Branch, Walter Reed Army Institute of Research, 503 Robert Grant Ave, Silver Spring, MD 20910, USA. ${ }^{3}$ Mahidol-Oxford Tropical Medicine Research Unit, Faculty of Tropical Medicine, Mahidol University, 420/6 Rajvithi Road, Bangkok 10400, Thailand. ${ }^{4}$ Centre for Tropical Medicine and Global Health, Nuffield Department of Clinical Medicine, University of Oxford, Old Road Campus, Roosevelt Drive, Oxford OX3 7FZ, UK. ${ }^{5}$ Pharmacology and Toxicology Unit, Faculty of Allied Health Sciences, 99 Mu 18 Thammasat University (Rangsit Campus), Klong Luang, Pathumthani 12121, Thailand. ${ }^{6}$ Armed Forces Pest Management Board, 2460 Linden Lane, Silver Spring, MD 20910, USA.

\section{Acknowledgements}

We thank all P. vivax blood donors. We would like to acknowledge the help of the AFRIMS Department of Entomology Malariology, Vector Biology \& Control, and Insectary section staff. This work was performed while Kevin Kobylinski held a National Research Council Fellowship. This work was supported by Grant OPP1095931 from the Bill \& Melinda Gates Foundation. Mahidol-Oxford Tropical Medicine Research Unit is supported by the Wellcome Trust, UK. The opinions or assertions contained herein are the private views of the authors, and are not to be construed as official, or as reflecting true views of the Department of the Army or the Department of Defense.

\section{Competing interests}

The authors declare that they have no competing interests.

\section{Publisher's Note}

Springer Nature remains neutral with regard to jurisdictional claims in published maps and institutional affiliations.

Received: 25 April 2017 Accepted: 28 June 2017

Published online: 07 July 2017

\footnotetext{
References

1. von Seidlein $L$, Dondorp A. Fighting fire with fire: mass antimalarial drug administrations in an era of antimalarial resistance. Expert Rev Anti Infect Ther. 2015:13:715-30.

2. Van Bortel W, Trung H, Hoi L, Ham N, Chut N, Luu N, et al. Malaria transmission and vector behaviour in a forested malaria focus in central Vietnam and the implications for vector control. Malar J. 2010;9:373.
} 
3. Trung H, van Bortel W, Sochantha T, Keokenchanh K, Briët O, Coosemans M. Behavioural heterogeneity of Anopheles species in ecologically different localities in Southeast Asia: a challenge for vector control. Trop Med Int Health. 2005;10:251-62.

4. Chaccour C, Rabinovich N, Slater H, Canavati S, Bousema T, Lacerda M, et al. Establishment of the ivermectin research for malaria elimination network: updating the research agenda. Malar J. 2015;14:243.

5. Kobylinski K, Deus K, Butters M, Hongyu T, Gray M, da Silva I, et al. The effect of oral anthelmintics on the survivorship and re-feeding frequency of anthropophilic mosquito disease vectors. Acta Trop. 2010;116:119-25.

6. Fritz M, Siegert P, Walker E, Bayoh M, Vulule J, Miller J. Toxicity of bloodmeals from ivermectin-treated cattle to Anopheles gambiae s.l. Ann Trop Med Parasitol. 2009;103:539-47.

7. Chaccour C, Lines J, Whitty C. Effect of ivermectin on Anopheles gambiae mosquitoes fed on humans: the potential of oral insecticides in malaria control. J Infect Dis. 2010;202:113-6.

8. Ouédraogo A, Bastiaens G, Tiono A, Guelbéogo W, Kobylinski K, Ouédraogo $A$, et al. Efficacy and safety of the mosquitocidal drug ivermectin to prevent malaria transmission after treatment: a double-blind randomized, clinical trial. Clin Infect Dis. 2015;60:357-65.

9. Derua Y, Kisinza W, Simonsen P. Differential effect of human ivermectin treatment on blood feeding Anopheles gambiae and Culex quinquefasciatus. Parasites Vectors. 2015;8:130.

10. Sylla M, Kobylinski K, Gray M, Chapman P, Sarr M, Rasgon J, Foy B. Mass drug administration of ivermectin in south-eastern Senegal reduces the survivorship of wild-caught, blood fed malaria vectors. Malar J. 2010;9:365.

11. Alout H, Krajacich B, Meyers J, Grubaugh N, Brackney D, Kobylinski K, et al. Evaluation of ivermectin mass drug administration for malaria transmission control across different West African environments. Malar J. 2014;13:417.

12. Kobylinski K, Sylla M, Chapman P, Sarr M, Foy B. Short report: ivermectin mass drug administration to humans disrupts malaria parasite transmission in Senegalese villages. Am J Trop Med Hyg. 2011;85:3-5.

13. Kobylinski K, Foy B, Richardson J. Ivermectin inhibits the sporogony of Plasmodium falciparum in Anopheles gambiae. Malar J. 2012;11:381.

14. Kobylinski K, Alout H, Foy B, Clements A, Adisakwattana P, Swierczewski $B$, Richardson J. Rationale for the coadministration of albendazole and ivermectin to humans for malaria parasite transmission control. Am J Trop Med Hyg. 2014;91:655-62.

15. Obsomer V, Defourny P, Coosemans M. The Anopheles dirus complex: spatial distribution and environmental drivers. Malar J. 2007:6:26.

16. Tisgratog R, Tananchai C, Juntarajumnong W, Tuntakom S, Bangs M, Corbel V, Chareonviriyaphap T. Host feeding patterns and preference of Anopheles minimus (Diptera: Culicidae) in a malaria endemic area of western Thailand: baseline site description. Parasites Vectors. 2012;5:114.

17. Rattanarithikul R, Konishi E, Linthicum K. Detection of Plasmodium vivax and Plasmodium falciparum circumsporozoite antigen in anopheline mosquitoes collected in southern Thailand. Am J Trop Med Hyg. 1996:54:114-21.

18. Thongsahuan S, Baimai V, Junkum A, Saeung A, Min G, Joshi D, et al. Susceptibility of Anopheles campestris-like and Anopheles barbirostris species complexes to Plasmodium falciparum and Plasmodium vivax in Thailand. Mem Inst Oswaldo Cruz. 2011:106:105-12.

19. Guzzo C, Furtek C, Porras A, Chen C, Tipping R, Clineschmidt C, et al. Safety, tolerability, and pharmacokinetics of escalating high doses of ivermectin in healthy adult subjects. J Clin Pharmacol. 2002;42:1122-33.

20. Suputtamongkol Y, Premasathian N, Bhumimuang K, Waywa D, Nilganuwong S, Karuphong E, et al. Efficacy and safety of single and double doses of ivermectin versus 7-day high dose albendazole for chronic strongyloidiasis. PLoS Negl Trop Dis. 2011;5:1044.

21. Awadzi K, Opoku N, Addy E, Quartey B. The chemotherapy of onchocerciasis. XIX: the clinical and laboratory tolerance of high dose ivermectin. Trop Med Parasitol. 1995;46:131-7.

22. Awadzi K, Attah S, Addy E, Opoku N, Quartey B. The effects of high-dose ivermectin regimens on Onchocerca volvulus in onchocerciasis patients. Trans R Soc Trop Med Hyg. 1999;93:189-94.

23. Gardon J, Boussinesq M, Kamgno J, Gardon-Wendel N, Duke BO. Effects of standard and high doses of ivermectin on adult worms of Onchocerca volvulus: a randomised controlled trial. Lancet. 2002;360:203-10.
24. Na-Bangchang K, Kietinun S, Pawa K, Hanpitakpong W, Na-Bangchang C, Lazdins J. Assessments of pharmacokinetic drug interactions and tolerability of albendazole, praziquantel and ivermectin combinations. Trans $R$ Soc Trop Med Hyg. 2006;100:335-45.

25. Jonsson E, Karlsson M. Xpose-an S-PLUS based population pharmacokinetic/pharmacodynamic model building aid for NONMEM. Comput Methods Programs Biomed. 1999:8:51-64.

26. Keizer R, van Benten M, Beijnen J, Schellens J, Huitema A. Pirana and PCluster: a modeling environment and cluster infrastructure for NONMEM. Comput Methods Programs Biomed. 2011;101:72-9.

27. Lindbom L, Ribbing J, Jonsson E. Perl-speaks-NONMEM (PsN)—a Perl module for NONMEM related programming. Comput Methods Programs Biomed. 2004;75:85-94.

28. Macey R, Oster G, Zahnley T, Gittelsohn M. Berkeley Madonna. Berkeley. 2000.

29. Phasomkusolsil S, Pantuwattana K, Tawong J, Khongtak W, Kertmanee Y, Monkanna N, et al. The relationship between wing length, blood meal volume, and fecundity for seven colonies of Anopheles species housed at the Armed Forces Research Institute of Medical Sciences, Bangkok, Thailand. Acta Trop. 2015;152:220-7.

30. Sattabongkot J, Maneechai N, Phunkitchar V, Eikarat N, Khuntirat B, Sirichaisinthop J, et al. Comparison of artificial membrane feeding with direct skin feeding to estimate the infectiousness of Plasmodium vivax gametocyte carriers to mosquitoes. Am J Trop Med Hyg. 2003;69:529-35.

31. Tainchum K, Kongmee M, Manguin S, Bangs M, Chareonviriyaphap T. Anopheles species diversity and distribution of the malaria vectors of Thailand. Trends Parasitol. 2015;31:109-19.

32. Zeng Z, Andrew N, Arison B, Luffer-Atlas D, Wang R. Identification of cytochrome P4503A4 as the major enzyme responsible for the metabolism of ivermectin by human liver microsomes. Xenobiotica. 1998;28:313-21.

33. Merck Research Laboratories. Stromectol new drug application. In: FDA Center for drug evaluation and research. 1996. http://www.accessdata. fda.gov/drugsatfda_docs/label/2009/050742s026lbl.pdf. Accessed 5 June 2015.

34. Bellinger A, Jafari M, Grant T, Zhang S, Slater H, Wegner E, et al. Oral, ultralong-lasting drug delivery: application toward malaria elimination goals. Sci Transl Med. 2016;8:157.

35. Chaccour C, Barrio A, Royo AG, Urbistondo DM, Slater H, Hammann F, Del Pozo J. Screening for an ivermectin slow-release formulation suitable for malaria vector control. Malar J. 2015:14:102.

36. Chaccour C, Abizanda G, Irigoyen Á, Del Pozo J. Pilot study of a slowrelease ivermectin formulation for malaria control in a pig model. Antimicrob Agents Chemother. 2017;61:02104-16.

37. Baraka O, Mahmoud B, Marschke C, Geary T, Homeida M, Williams J. Ivermectin distribution in the plasma and tissues of patients infected with Onchocerca volvulus. Eur J Clin Pharmacol. 1996;50:407-10.

38. El-Tahtawy A, Glue P, Andrews E, Mardekian J, Amsden G, Knirsch C. The effect of azithromycin on ivermectin pharmacokinetics-a population pharmacokinetic model analysis. PLoS Negl Trop Dis. 2008;2:236.

39. Holford N. A size standard for pharmacokinetics. Clin Pharmacokinet. 1996:30:329-32.

40. Anderson B, Holford N. Mechanism-based concepts of size and maturity in pharmacokinetics. Ann Rev Pharmacol Toxicol. 2008;48:303-32.

41. Smit M, Ochomo E, Aljayyoussi G, Kwambai T, Abong'o B, Bayoh N, et al. Efficacy and safety of high-dose ivermectin for reducing malaria transmission (IVERMAL): protocol for a double-blind, randomized, placebo-controlled, dose-finding trial in Western Kenya. JMIR Res Protoc. 2016:5:213.

42. Ramaiah K, Das P, Vanamail P, Pani S. Impact of 10 years of diethylcarbamazine and ivermectin mass administration on infection and transmission of lymphatic filariasis. Trans R Soc Trop Med Hyg. 2007;101:555-63.

43. Cooper P, Awadzi K, Ottesen E, Remick D, Nutman T. Eosinophil sequestration and activation are associated with the onset and severity of systemic adverse reactions following the treatment of onchocerciasis with ivermectin. J Infect Dis. 1999;179:738-42.

44. Martin-Prevel Y, Cosnefroy J, Tshipamba P, Ngari P, Chodakewitz J, Pinder $M$. Tolerance and efficacy of single high-dose ivermectin for the treatment of loiasis. Am J Trop Med Hyg. 1993;48:186-92. 
45. Ismail M, Weil G, Jayasinghe K, Premaratne U, Abeyewickreme W, Rajaratnam $\mathrm{H}$, et al. Prolonged clearance of microfilaraemia in patients with bancroftian filariasis after multiple high doses of ivermectin or diethylcarbamazine. Trans R Soc Trop Med Hyg. 1996;90:684-8.

46. Ismail M, Jayakody R, Weil G, Nimalan N, Jayasinghe K, Abeyewickrema W, et al. Efficacy of single dose combinations of albendazole, ivermectin and diethylcarbamazine for the treatment of bancroftian filariasis. Trans R Soc Trop Med Hyg. 1998;92:94-7.

47. Ismail M, Jayakody R, Weil G, Fernando D, de Silva G, Balasooriya W. Longterm efficacy of single-dose combinations of albendazole, ivermectin and diethylcarbamazine for the treatment of bancroftian filariasis. Trans $R$ Soc Trop Med Hyg. 2001;95:332-5.

48. Dembele B, Coulibaly Y, Dolo H, Konate S, Coulibaly S, Sanogo D, et al. Use of high-dose, twice-yearly albendazole and ivermectin to suppress Wuchereria bancrofti microfilarial levels. Clin Infect Dis. 2010;51:1229-35.

49. Bockarie M, Alexander N, Hyun P, Dimber Z, Bockarie F, Ibam E, et al. Randomised community-based trial of annual single-dose diethylcarbamazine with or without ivermectin against Wuchereria bancrofti infection in human beings and mosquitoes. Lancet. 1998;351:162-8.

50. Kazura J, Greenberg J, Perry R, Weil G, Day K, Alpers M. Comparison of single-dose diethylcarbamazine and ivermectin for treatment of bancroftian filariasis in Papua New Guinea. Am J Trop Med Hyg. 1993;49:804-11.

51. Cartel J, Moulia-Pelat J, Glaziou P, Nguyen L, Chanteau S, Roux J. Results of a safety trial on single-dose treatments with $400 \mathrm{mcg} / \mathrm{kg}$ of ivermectin in bancroftian filariasis. Trop Med Parasitol. 1992;43:263-6.

52. Glaziou P, Moulia-Pelat J, Nguyen L, Chanteau S, Martin P, Cartel J. Doubleblind controlled trial of a single dose of the combination ivermectin 400 micrograms $/ \mathrm{kg}$ plus diethylcarbamazine $6 \mathrm{mg} / \mathrm{kg}$ for the treatment of bancroftian filariasis: results at six months. Trans R Soc Trop Med Hyg. 1994;88:707-8.

53. Moulia-Pelat J, Glaziou P, Nguyen L, Chanteau S, Pilchart R, Beylier I, et al. Ivermectin 400 micrograms/kg: long-term suppression of microfilariae in bancroftian filariasis. Trans R Soc Trop Med Hyg. 1994;88:107-9.

54. Moulia-Pelat J, Nguyen L, Glaziou P, Chanteau S, Ottesen E, Cardines R, et al. Ivermectin plus diethylcarbamazine: an additive effect on early microfilarial clearance. Am J Trop Med Hyg. 1994;50:206-9.

55. Moulia-Pelat J, Glaziou P, Nguyen L, Cartel J. Single doses of ivermectin 400 micrograms/kg: the most effective dosage in bancroftian filariasis. Southeast Asian J Trop Med Public Health. 1995;26:124-7.

56. Nguyen L, Moulia-Pelat J, Cartel J. Control of bancroftian filariasis in an endemic area of Polynesia by ivermectin 400 micrograms $/ \mathrm{kg}$. Trans R Soc Trop Med Hyg. 1996;90:689-91.

57. Nguyen L, Moulia-Pelat J, Glaziou P, Martin P, Cartel J. Advantages of ivermectin at a single dose of 400 micrograms $/ \mathrm{kg}$ compared with 100 micrograms/kg for community treatment of lymphatic filariasis in Polynesia. Trans R Soc Trop Med Hyg. 1994;88:461-4.

58. Moulia-Pelat J, Nguyen L, Hascoët H, Nicolas L. Combinations of ivermectin and diethylcarbamazine for improved control of lymphatic filariasis. Parasite. 1996;3:45-8.

59. Dreyer G, Coutinho A, Miranda D, Norões J, Rizzo J, Galdino E, et al. Treatment of bancroftian filariasis in Recife, Brazil: a two-year comparative study of the efficacy of single treatments with ivermectin or diethylcarbamazine. Trans R Soc Trop Med Hyg. 1995;89:98-102.

60. Dreyer G, Norões J, Amaral F, Nen A, Medeiros Z, Coutinho A, Addiss D. Direct assessment of the adulticidal efficacy of a single dose of ivermectin in bancroftian filariasis. Trans R Soc Trop Med Hyg. 1995;89:441-3.

61. Dreyer G, Addiss D, Norões J, Amaral F, Rocha A, Coutinho A. Ultrasonographic assessment of the adulticidal efficacy of repeat high-dose ivermectin in bancroftian filariasis. Trop Med Int Health. 1996;1:427-32.

62. Dreyer G, Addiss D, Santos A, Figueredo-Silva J, Norões J. Direct assessment in vivo of the efficacy of combined single-dose ivermectin and diethylcarbamazine against adult Wuchereria bancrofti. Trans R Soc Trop Med Hyg. 1998;92:219-22.

63. Addiss D, Eberhard M, Lammie P, McNeeley M, Lee S, McNeeley D, Spencer H. Comparative efficacy of clearing-dose and single high-dose ivermectin and diethylcarbamazine against Wuchereria bancrofti microfilaremia. Am J Trop Med Hyg. 1993;48:178-85.

64. Addiss D, Beach M, Streit T, Lutwick S, LeConte F, Lafontant J, et al. Randomised placebo-controlled comparison of ivermectin and albendazole alone and in combination for Wuchereria bancrofti microfilaraemia in Haitian children. Lancet. 1997;350:480-4.
65. Chosidow O, Giraudeau B, Cottrell J, Izri A, Hofmann R, Mann S, Burgess I. Oral ivermectin versus malathion lotion for difficult-to-treat head lice. N Engl J Med. 2010;362:896-905.

66. Merck, Sharp \& Dohme (France). Mectizan Package Insert. In: Base de données publique des médicaments. 2014. http://base-donnees-publique.medicaments.gouv.fr/affichageDoc.php? specid=61350360\&typedo $\mathrm{c}=\mathrm{N}$. Accessed 12 Dec 2015.

67. Bousema T, Drakeley C. Epidemiology and infectivity of Plasmodium falciparum and Plasmodium vivax gametocytes in relation to malaria control and elimination. Clin Microbiol Rev. 2011;24:377-410.

68. Pooda H, Rayaisse J-B, de Sale Hien D, Lefèvre T, Yerbanga S, Bengaly Z, et al. Administration of ivermectin to peridomestic cattle: a promising approach to target the residual transmission of human malaria. Malar J. 2015;14:496.

69. Mahmood F, Walters L, Guzman H, Tesh R. Effect of ivermectin on the ovarian development of Aedes aegypti (Diptera: Culicidae). J Med Entomol. 1991;28:701-7.

70. Seaman J, Alout H, Meyers J, Stenglein M, Dabiré R, Lozano-Fuentes S, et al. Age and prior blood feeding of Anopheles gambiae influences their susceptibility and gene expression patterns to ivermectin-containing blood meals. Malar J. 2015;16:797.

71. Alout H, Foy B. Ivermectin: a complimentary weapon against the spread of malaria? Expert Rev Anti Infect Ther. 2017;15:231-40.

72. Cirimotich C, Dong Y, Clayton AM, Sandiford SL, Souza-Neto JA, Mulenga M, Dimopoulos G. Natural microbe-mediated refractoriness to Plasmodium infection in Anopheles gambiae. Science. 2011;332:855-8.

73. Burg R, Stapley E. Isolation and characterization of the producing organism. In: Campbell WC, editor. Ivermectin and abamectin. New York: Springer; 1989. p. 24-32.

74. Guo H, Ren B, Dai H, Dai S, Zhang Y, Liu Y, Cao B, Zhang L. Reversal of meticillin resistance in Staphylococcus aureus by the anthelmintic avermectin. Int J Antimicrob Agents. 2014;44:274-6.

75. Pettengill M, Lam V, Ollawa I, Marques-da-Silva C, Ojcius D. Ivermectin inhibits growth of Chlamydia trachomatis in epithelial cells. PLoS ONE. 2012;7:48456.

76. Lim L, Vilchèze C, Ng C, Jacobs WJ, Ramón-García S, Thompson C. Anthelmintic avermectins kill Mycobacterium tuberculosis, including multidrugresistant clinical strains. Antimicrob Agents Chemother. 2013;57:1040-6.

77. Rodgers F, Gendrin M, Wyer C, Christophides G. Microbiota-induced peritrophic matrix regulates midgut homeostasis and prevents systemic infection of malaria vector mosquitoes. PLoS Pathog. 2017;13:1006391.

78. Hotez P, Bottazzi M, Strych U, Chang L, Lim Y, Goodenow M, AbuBakar S. Neglected tropical diseases among the Association of Southeast Asian Nations (ASEAN): overview and update. PLoS Negl Trop Dis. 2015;9:0003575.

79. Khieu V, Schär F, Forrer A, Hattendorf J, Marti H, Duong S, et al. High prevalence and spatial distribution of Strongyloides stercoralis in rural Cambodia. PLoS Negl Trop Dis. 2014;8:2854.

80. Vonghachack Y, Sayasone S, Bouakhasith D, Taisayavong K, Akkavong K, Odermatt P. Epidemiology of Strongyloides stercoralis on Mekong islands in southern Laos. Acta Trop. 2015;141:289-94.

81. Khieu V, Hattendorf J, Schär F, Marti H, Char M, Muth S, Odermatt P. Strongyloides stercoralis infection and re-infection in a cohort of children in Cambodia. Parasitol Int. 2014;63:708-12.

82. Okeibunor J, Amuyunzu-Nyamongo M, Onyeneho N, Tchounkeu Y, Manianga C, Kabali A, Leak S. Where would I be without ivermectin? Capturing the benefits of community-directed treatment with ivermectin in Africa. Trop Med Int Health. 2011;16:608-21.

83. Eisele T, Bennett A, Silumbe K, Finn T, Chalwe V, Kamuliwo M, et al. Short-term impact of mass drug administration with dihydroartemisinin plus piperaquine on malaria in Southern Province Zambia: a clusterrandomized controlled trial. J Infect Dis. 2016;214:1831-9.

84. Slater H, Walker P, Bousema T, Okell L, Ghani A. The potential impact of adding ivermectin to a mass treatment intervention to reduce malaria transmission: a modelling study. J Infect Dis. 2014;210:1972-80. 